\title{
PET/CT for endocarditis in the ACC/AHA 2020 valve guidelines: Ready for prime time?
}

We read with great interest the long awaited $2020 \mathrm{ACC} /$ AHA guidelines for valvular heart disease management. ${ }^{1}$ Although we applaud the introduction of ${ }^{18} \mathrm{~F}$ fluorodeoxyglucose positron emission tomography/computed tomography $\left({ }^{18} \mathrm{FDG}-\mathrm{PET} / \mathrm{CT}\right)$ in the management algorithm for patients with possible endocarditis by the Modified Duke Criteria, we caution against its broader use in native valve endocarditis. Contemporary literature best supports the use of ${ }^{18}$ FDG$\mathrm{PET} / \mathrm{CT}$ for prosthetic valve or cardiac implantable electronic device (CIED, at least 3-months from implantation) endocarditis (pooled sensitivity $72 \%$ to $86 \%$, specificity $83 \%$ to $84 \%$ in a recent meta-analysis), but not native valve endocarditis (pooled sensitivity only $31 \%$, despite specificity $98 \%$ ). ${ }^{2}$ Reasons for the latter include more fibrotic and less inflammatory lesions in native valve endocarditis, under-utilization of ECG-gating during scans, limited spatial resolution, and insufficient metabolic preparation with diffuse myocardial uptake masking valve inflammation. Radio-labelled white blood cell single-photon emission computed tomography (WBC-SPECT)/CT enhances specificity of "inflammation" imaging and may be utilized for this purpose. ${ }^{3}$

The revised guidelines appropriately incorporate new imaging modalities in the management of endocarditis. However, the expansion of ${ }^{18}$ FDG-PET/CT utilization beyond prosthetic valve/CIED infection detection and without a time horizon from CIED insertion may confuse the diagnosis and result in unwarranted high risk therapies. A nuanced approach with ${ }^{18}$ FDG$\mathrm{PET} / \mathrm{CT}$ as a highly sensitive test late after prosthetic valve/CIED insertion and (WBC-SPECT)/CT as a high specific test early after prosthetic valve/device is warranted. ${ }^{2,3}$ Therefore, we propose distinctions between native and prosthetic valve/CIED endocarditis, and consideration of timing from surgery. Limited diagnostic accuracy of ${ }^{18} \mathrm{FDG}-\mathrm{PET} / \mathrm{CT}$ in native valve endocarditis should be emphasized. In suspected prosthetic valve/CIED infection where echocardiography is negative or inconclusive, ${ }^{18}$ FDG-PET/CT should be performed because of excellent sensitivity and wide availability for diagnosis and evaluating perivalvular complications. If increased specificity is required, which may be especially relevant soon after surgery, (WBCSPECT)/CT can provide confirmation of these diagnoses.

Wael A. Jaber, MD, ${ }^{a}$ Tom Kai Ming Wang, MBChB, $M D($ res $),{ }^{a}$ Paul C. Cremer, $M D^{a}$

${ }^{a}$ Section of Cardiovascular Imaging, Heart, Vascular and Thoracic Institute, Cleveland Clinic, 9500 Euclid Avenue, Main Campus J15, Cleveland, $\mathrm{OH} 44195$, USA

Funding statements

None to declare for all authors.

Disclosures Authors Dr Jaber, Dr Wang and Dr Cremer with no conflicts of interest to declare

\section{References}

1. Otto CM, Nishimura RA, Bonow RO, Carabello BA, Erwin JP 3rd, Gentile F et al (2021) 2020 ACC/AHA guideline for the management of patients with Valvular Heart Disease: A Report of the American College of Cardiology/American Heart Association Joint Committee on Clinical Practice Guidelines. J Am Coll Cardiol 77(4):e25-e197

2. Wang TKM, Sánchez-Nadales A, Igbinomwanhia E, Cremer P, Griffin B, Xu B (2020) Diagnosis of infective endocarditis by subtype using (18)F-fluorodeoxyglucose positron emission tomography/computed tomography: A contemporary meta-analysis. Circ Cardiovasc Imaging 13(6): $\mathrm{e} 010600$

3. Erba PA, Conti U, Lazzeri E, Sollini M, Doria R, De Tommasi SM et al (2012) Added value of 99mTc-HMPAO-labeled leukocyte SPECT/CT in the characterization and management of patients with infectious endocarditis. J Nucl Med 53(8):1235-1243

doi:10.1007/s12350-021-02748-x

Publisher's Note Springer Nature remains neutral with regard to jurisdictional claims in published maps and institutional affiliations. 